\title{
Channel Quality Indicator Feedback in Long Term Evolution (LTE) System
}

\author{
Maina Ibrahim \\ Wireless Communication Centre (WCC), Faculty of Electrical Engineering, Universiti Teknologi Malaysia \\ (UTM), Malaysia
}

\begin{abstract}
LTE is a $3 G P P$ evolution of UMTS that is envisaged to provide high data rate and low latency in order to satisfy the data rate requirement of current high data rate applications. To achieve this and guaranty transmission at all times, LTE system adjust transmission parameters like PMI, RI and CQI dynamically. Accomplished via feedback from UE, the feedbacks are set after every transmission time interval based on the measured link quality as adjudged by post detection SINR at the receiver. This paper examined CIQ feedback in LTE using Vienna LTE Link Level Simulator for ITU-T Pedestrian A and Vehicular A channels for different receiver and channel knowledge. It was seen that CQI feedback brings performance improvement for the different scenarios studied. In all, Pedestrian A channel showed better performance compared to Vehicular A channel.
\end{abstract}

Keywords: Channel quality indicator (CQI), Downlink, Feedback, LTE, SINR

\section{Introduction}

The evolution of Universal Mobile Telecommunication System (UMTS) by the $3^{\text {rd }}$ Generation Partnership Project (3GPP) is named Long Term Evolution (LTE). Its main targets are: data rates up to $100 \mathrm{Mbit} / \mathrm{s}$ on downlink and up to 50Mbit/s on uplink, support many users, reduce latency, cost efficient and support only packet-switched services [1]. By these, it will meet the data rate requirement of data hungry devices like smartphones, tablets, cloud computing etc. To sustain its service and guarantee transmission at all times, the LTE system is designed to adjust its transmission parameters such as Channel Quality Indicator (CQI), Rank Indicator (RI) and Precoding Matrix Indicator (PMI) dynamically to match the channel condition in what is known as Link Adaptation [2]. This as opposed to hitherto worst case scenario configuration. The mentioned targets are achieved by employing Orthogonal Frequency Division Multiplexing Access (OFDMA) to increase significantly spectral efficiency [3], Multiple Input Multiple Output (MIMO) antennas taking advantage of multipath to enhance the radio channel capacity at no extra cost of spectrum [4]. There is also spectrum flexibility in LTE for which it can operate at 1.4, 3, 5, 10, 15 and 20MHz bandwidths [5].

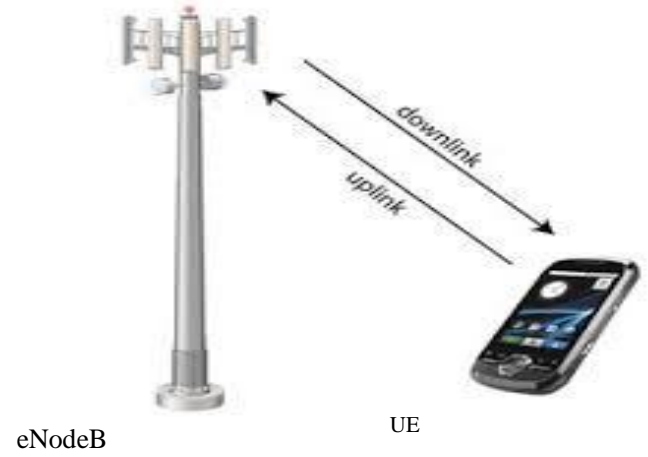

Figure 1. LTE Downlink/Uplink

In LTE, the downlink transmission is the transmission from evolved NodeB(eNB) to mobile terminal or user equipment (UE). Uplink in turn is from UE to eNB, see Fig. 1. OFDM is used on downlink and Single Carrier FDMA (SC-FDMA) on the uplink. Time Division Duplex (TDD) and Frequency Division Duplex (FDD) frame structures are also defined for LTE. By OFDM, the used bandwidth is divided into a large number of mutual orthogonal narrowband subcarriers. Every subcarrier is singly modulated by a low rate data stream. Using OFDMA these subcarriers can be allocated between the multiple users. Each user is given a specific time/ frequency resources. Fundamentally, data channels are shared channels. Meaning after each transmission time interval (TTI) of $1 \mathrm{~ms}$, a new scheduling is made reassigning the time/frequency resources again to the users. This technique ensures very high spectral efficiency allowing for the exploitation of the variations in both frequency and time domains. Physical Resource Blocks (PRB) is the unit for data allocation to the UEs. Integer 
multiples of PRB are allocated to UE. A PRB constitute 12 subcarriers in frequency grid. The subcarriers has a spacing of $15 \mathrm{kHz}$. Hence the bandwidth of a PRB is $180 \mathrm{kHz}$. For LTE bandwidths, the allocated number of PRBs are shown in Table 1.

Table 1. Distribution of Resource Blocks for LTE Bandwidths

\begin{tabular}{|l|l|l|l|l|l|l|}
\hline Bandwidth(MHz) & 1.4 & 3 & 5 & 10 & 15 & 20 \\
\hline Number of RBs & 6 & 15 & 25 & 50 & 75 & 100 \\
\hline
\end{tabular}

The scheduling and link adaptation task are fulfilled in the eNB by a feedback from the UE. The frequency of UE feedback depend on the set periodicity. The feedback may include CQI, PMI and RI. The CQI carries the suitable transmission rate which is used for resource allocation and link adaptation by the eNB for the downlink. It is used for dynamic utilising of the available system resources efficiently. That is, selecting the appropriate Modulation order and Coding Scheme (MCS) that matches current link quality that is usually estimated from Signal to Interference plus Noise Ratio (SINR) measured at UE. In the feedback, number of antennas, type of receiver used etc. are taken into account. The feedback signalled is always the one that can achieve a target block error ratio (BLER) $\leq 0.1$ which is the typical value for mobile communication [6]. PMI and RI are for MIMO pre-processing and ensures that the correct rank and precoders that will maximise throughput are set. The feedback granularity for PMI and CQI in LTE ranges from one value per PRB and or one for the whole system bandwidth [7].

In this paper, attention was focussed on CQI feedback only. The feedback performance under different channel types and detection techniques with a particular antenna configuration were examined. The remaining discussion in this paper is organised as follows: The theory for LTE downlink transmission was briefly highlighted in Section II while CQI feedback computation is treated in Section III. Simulation results are shown in Section IV and finally conclusion is drawn in Section V.

\section{Theory}

Consider an LTE system with $N_{R}$ receive and $N_{T}$ transmit antennas. The frequency selective channels are converted to a finite number of frequency flat narrow band channels using OFDM. For these narrow band channels or subcarriers, the input output relationship can be expressed as:

$$
\mathbf{y}=\mathbf{H x}+\mathbf{z}
$$

where $\mathbf{x} \in \mathrm{C}^{N_{R} \times 1}$ represent the transmit and $\mathbf{y} \in \mathrm{C}^{N_{R} \times 1}$ the receive signal vectors. $\mathbf{H} \in \mathrm{C}^{N_{R} \times N_{T}}$ with entries $h_{i, j}$ (gain between $i^{\text {th }}$ and $j^{\text {th }}$ antenna at the transmitter and antenna at receiver) denote the channel matrix. Whence, $i=1,2, \ldots, N_{T}$ and $j=1,2, \ldots, N_{R} . z \square \mathcal{C N}\left(0, \sigma_{z}^{2} \cdot \mathbf{I}\right)$ is white Gaussian noise with variance $\sigma_{z}^{2}$.

Linear detectors has been successfully employed at the receive side for detection. To perform the detection, the transmitted signals are treated as interference with the exception of the desired stream of the target antenna. This means that apart from the needed stream, others which are considered as interferes are minimised. So, a weight matrix $\mathbf{W}$ is used to invert the effect of the channel yielding:

$$
\mathbf{y}=\mathbf{H W x}+\mathbf{z}
$$

A linear equalizer then filters $\mathbf{y}$ and the post equalisation signal is given by:

$$
\tilde{\mathbf{y}}=\mathbf{F H W x}+\mathbf{F z}
$$

with $\mathbf{F} \in \mathrm{C}^{L \times N_{R}}$ and $\mathrm{L}$ number of transmission layers. By rendering these, the post processing SINR for a subcarrier $i$ and spatial layer $l$ by setting $\mathbf{K}=\mathbf{F H W}$ can be evaluated from [6]:

$$
\operatorname{SINR}_{l, i}=\frac{|\mathbf{K}(l, l)|^{2}}{\sum_{i \neq l}|\mathbf{K}(l, i)|^{2}+\sigma_{z}^{2} \sum_{i} \mathbf{F}(l, i)}
$$

\section{CQI Feedback}

LTE support wideband and subband reporting of CQI [3]. Reporting periodicity is controlled by eNB and can be periodic or aperiodic depending on traffic pattern. The wideband CQI mask out frequency selective SINR variation and selective scheduling is supported with subband CQI reporting. In wideband CQI reporting, one value of CQI is reported for the whole system bandwidth by UE. Whereas in subband reporting, UE reports one value of CQI for the whole system bandwidth with an additional CQI value for every subband. A subband 
consist of $n$ contagious PRBs with $2 \leq n \leq 8$ [8]. 3GPP has standardised 15 MCS values for LTE. Table 2 shows the associated CQI index and the SINR switching threshold with 10\% BLER for the corresponding MCS [9].

Table 2. MCS Feedback Table

\begin{tabular}{|c|c|c|c|c|}
\hline $\begin{array}{c}\text { CQI } \\
\text { Index }\end{array}$ & $\begin{array}{c}\text { Modulation } \\
\text { Order }\end{array}$ & $\begin{array}{c}\text { Code Rate } \\
\times 1024\end{array}$ & $\beta$ & $\begin{array}{c}\text { SINR threshold } \\
(\mathrm{dB})\end{array}$ \\
\hline 0 & \multicolumn{5}{|c|}{ No transmission } \\
\hline 1 & QPSK & 78 & 1.00 & -9.478 \\
\hline 2 & QPSK & 120 & 1.40 & -6.658 \\
\hline 3 & QPSK & 193 & 1.40 & -4.098 \\
\hline 4 & QPSK & 308 & 1.48 & -1.798 \\
\hline 5 & QPSK & 449 & 1.50 & 0.399 \\
\hline 6 & QPSK & 602 & 1.62 & 2.424 \\
\hline 7 & 16QAM & 378 & 3.10 & 4.489 \\
\hline 8 & 16QAM & 490 & 4.32 & 6.367 \\
\hline 9 & 16QAM & 616 & 5.37 & 8.456 \\
\hline 10 & 64QAM & 466 & 7.71 & 10.266 \\
\hline 11 & 64QAM & 567 & 15.5 & 12.218 \\
\hline 12 & 64QAM & 666 & 19.6 & 14.122 \\
\hline 13 & 64QAM & 772 & 24.7 & 15.849 \\
\hline 14 & 64QAM & 873 & 27.6 & 17.786 \\
\hline 15 & 64QAM & 948 & 28.0 & 19.809 \\
\hline
\end{tabular}

The general rule for the feedback is as follows: The downlink channel quality is measured by post equalisation signal to noise ratios of the resources of interest (subcarriers of the scheduled PRBs) at the receiver. So, if $\gamma_{i}$ are these SINRs, to perform the feedback an average value representing the link quality is determined. A variety of compression techniques exist but the 3GPP favoured Exponential SINR Mapping (ESM) [6]. For this work, Effective Exponential SINR Mapping (EESM) is used. The technique compress the SINRs into a single value equivalent to a SINR value of a Single Input Single Output (SISO) Additive White Gaussian Noise (AWGN) channel. It is mathematically expressed as:

$$
S N R_{e f f}=-\beta \log \left(\frac{1}{N} \sum_{i=1}^{N} \exp \left(-\frac{\gamma_{i}}{\beta}\right)\right)
$$

where $N$ is the number of the resources of interest. $\beta$ is a calibration factor that is MCS dependant [10]. Its typical values are given in Table 2. With this, the BLER performance of the equivalent AWGN channel is approximated to be same as that of the original OFDM system [11]. The SINR is then used with an offline generated SINR - BLER curves under AWGN channel to estimate the corresponding BLERs and the throughput is evaluated from:

$$
T_{i}=R_{i}\left(1-B L E R\left(\operatorname{SINR}_{e f f}\right) \log _{2} M_{i}\right.
$$

With $T_{i}, R_{i}$ and $M_{i}$ being the corresponding throughput, code rate and modulation order respectively. The CQI value that is fed back is the one that yield the highest value of $T$ with BLER $\leq 0.1$ for the equivalent SISO AWGN channel.

\section{Simulation Results}

Simulation results carried out using Vienna LTE Link Level Simulator [12] for Pedestrian A(PedA) and Vehicular A(VehA) channels with $2 \times 1$ MIMO configuration are presented here. Soft Sphere Detection (SSD), Zero Forcing (ZF) receivers were considered with minimum mean squared error (MMSE) estimated and perfect channel knowledge. The antennas were considered uncorrelated and the feedback is fed with zero delay. The user occupies the whole bandwidth. 1000 channel realizations were simulated for a SNR value. The simulation

Table 3. Simulation Parameters

\begin{tabular}{|l|l|}
\hline Parameter & Value \\
\hline System Bandwidth & $1.4 \mathrm{MHz}$ \\
\hline Number of Subcarriers & 72 \\
\hline Feedback Delay & 0 TTI \\
\hline Channel Model & ITU-T VehA, ITU-T PedA \\
\hline Antenna Configurations & 2 transmit, 1 receive(2 x 1) \\
\hline Receiver & Zero Forcing(ZF), Soft Sphere Detection(SSD) \\
\hline Feedback Granularity & Full bandwidth \\
\hline Channel estimator & Perfect Channel Knowledge, Estimated Channel(MMSE) \\
\hline
\end{tabular}


parameters are summarised in Table 3. Additionally, a single value of CQI is reported for the whole system bandwidth with the channel averaged over an RB before the SINR is calculated.

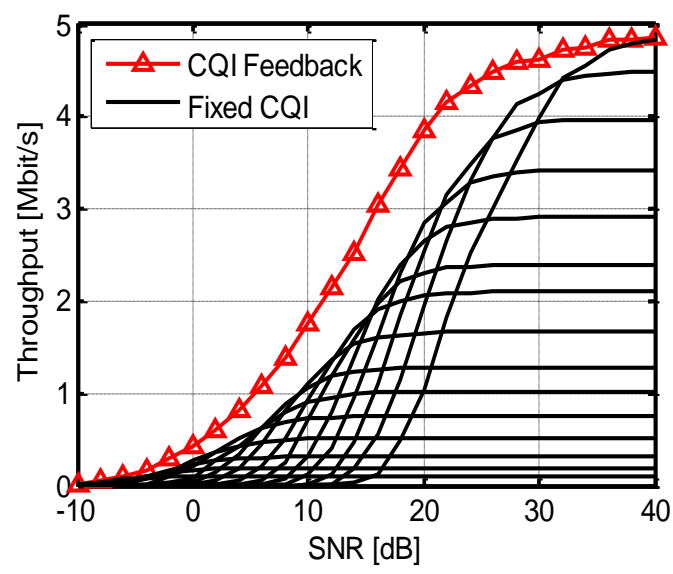

Figure 2. Throughput versus SNR for 2 x 1 VehA channel.

Fig. 2 depicts the result for throughput as a function of signal to noise ratio for a $2 \times 1$ VehA channel with ZF receiver and perfect knowledge of the channel when CQI feedback is activated and for fixed CQI's. The red diamond marked line shows when CQI feedback is activated while the black solid lines is when fixed CQI is used. The solid line with smallest throughput correspond to CQI 1 while the one with the highest throughput to CQI 15. Performance improvement with CQI feedback can clearly be seen. Throughput improvement of up to $1.01 \mathrm{Mbit} / \mathrm{s}$ over fixed CQI was possible. Similar trend of result was observed for estimated channel knowledge case and also with SSD receiver.

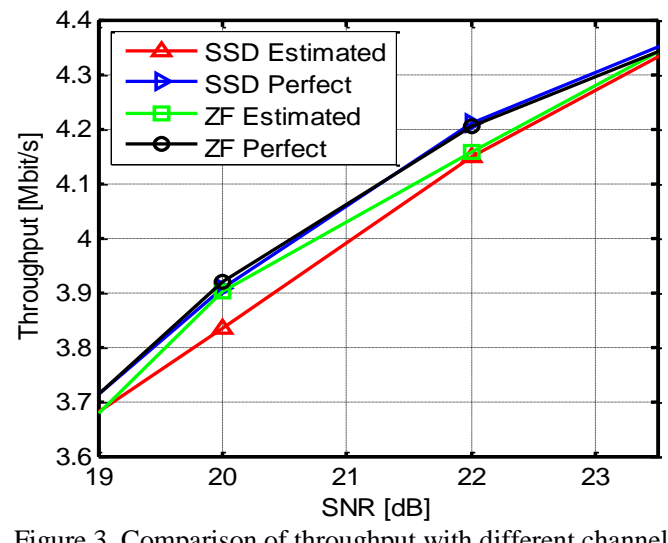

Figure 3. Comparison of throughput with different channel knowledge and receivers for 2 x 1 PedA channel.

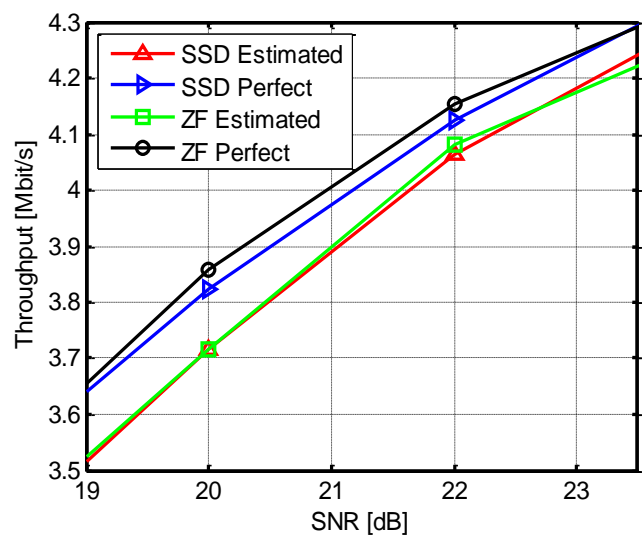

Figure 4. Comparison of throughput with different channe knowledge and receivers for 2 x 1 VehA channel.

Throughput as a function of transmit energy to noise power spectral density for $2 \times 1$ PedA channel employing SSD and ZF receivers for both perfect and estimated channel knowledge are shown in Fig. 3. The performance are almost same for SSD and ZF in the case of perfect channel knowledge. Compared to the estimated channel knowledge case, the performance is better with perfect channel knowledge. ZF with estimated channel shows inconsistent behaviour compared to the rest. A throughput improvement of up to $0.83 \mathrm{Mbit} / \mathrm{s}$ can be achieved between the two extreme scenarios. In Fig. 4, the same type of result is shown for VehA. It can be observed that perfect channel case still performed better than estimated channel case. In this situation unlike the case for PedA, SSD perfect has underperformed ZF perfect and the estimated channel case gave almost same result. Throughput difference of up to $0.142 \mathrm{Mbit} / \mathrm{s}$ between the two extreme cases is observed. 


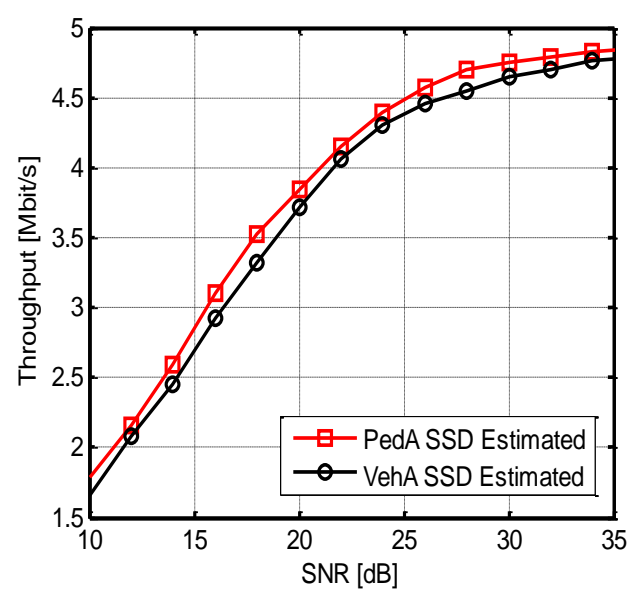

Figure 5. Throughput comparison for PedA/VehA channels with SSD receiver estimated channel knowledge.

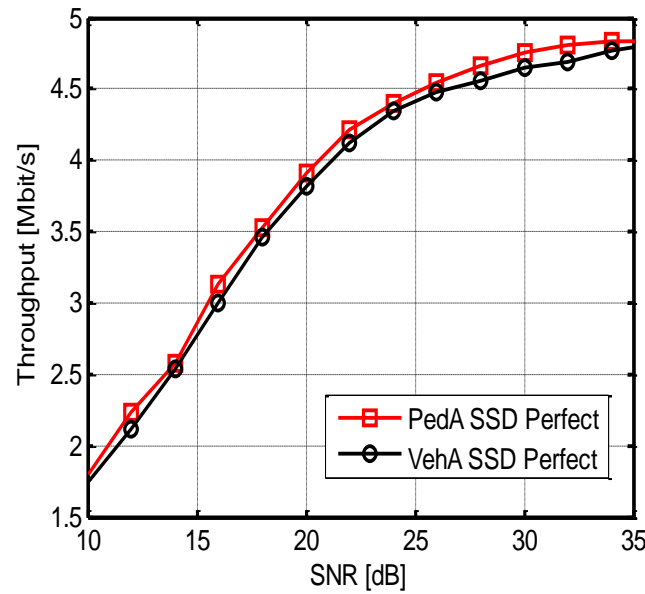

Figure 6. Throughput comparison for PedA/VehA channels with SSD receiver perfect channel knowledge.

Fig. 5 to 8 are the results for PedA/VehA SSD with estimated, perfect; PedA/VehA ZF with estimated, perfect channel knowledge's respectively. In all, the performance has same trend with PedA channel yielding better result in all compared to VehA.

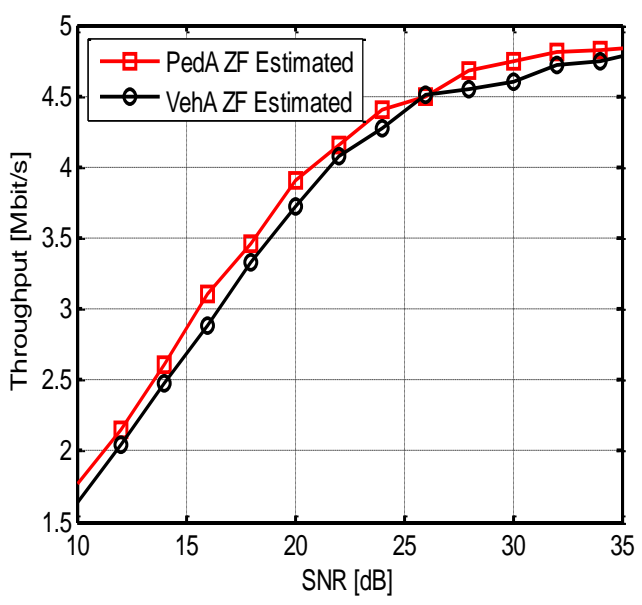

Figure 7. Throughput comparison for PedA/VehA channels with ZF receiver estimated channel knowledge.

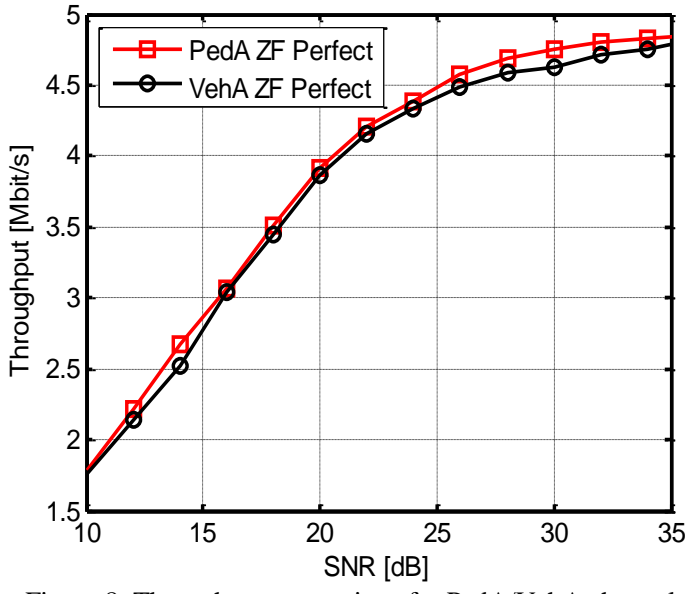

Figure 8. Throughput comparison for PedA/VehA channels with ZF receiver perfect channel knowledge.

\section{Conclusion}

In this paper, CQI feedback for PedA and VehA $2 \times 1$ MIMO LTE system with SSD and ZF receivers for perfect and estimated channel knowledge were examined. The CQI value which is valuable for link adaptation was derived from the instantaneous channel quality measured at the receive side by calculating the post detection SINR. With Vienna LTE Link Level Simulator as a test platform, performance improvement with CQI feedback is demonstrated. Generally for the cases under investigation, the case with perfect channel knowledge performed better compared with estimated channel case. It was also seen that with SSD, ZF receivers for perfect and estimated knowledge PedA channel shows better performance compared to VehA.

\section{References}

[1] R. Moray, LTE and the Evolution to $4 G$ Wireless: Design and Measurement Challenges. Great Britain: John Wiley \& Sons Ltd, 2009.

[2] H. Congzheng, S. Armour, A. Doufexi, N. Kah Heng, and J. McGeehan, "Link Adaptation Performance Evaluation for a MIMOOFDM Physical Layer in a Realistic Outdoor Environment," in Vehicular Technology Conference, 2006. VTC-2006 Fall. 2006 IEEE 64th, 2006, pp. 1-5.

[3] S. Stefania, T. Issam, and B. Matthew, LTE - The UMTS Long Term Evolution: From Theory to Practice. Great Britain: John Wiley \& Sons Ltd, 2009 .

[4] E. Biglieri, R. Calderbank, A. Constantinides, A. Goldsmith, A. Paulraj, and H. V. Poor, MIMO Wireless Communications. United Kingdom: Cambridge University Press, 2007.

[5] 3GPP TR 25.913, "Requirements for Evolved UTRA(E-UTRA) and Evolved UTRAN(E-UTRAN)," (Release 8), v8.0.0, 2009.

[6] S. Schwarz, C. Mehlfuhrer, and M. Rupp, "Calculation of the spatial preprocessing and link adaption feedback for 3GPP UMTS/LTE," in Wireless Advanced (WiAD), 2010 6th Conference on, 2010, pp. 1-6. 
[7] 3GPP TR 36.213, "LTE; Evolved Universal Terrestrial Radio Access(E-UTRA); Physical Layer Procedures," (Release 8), v8.8.0, 2009.

[8] S. N. Donthi and N. B. Mehta, "An Accurate Model for EESM and its Application to Analysis of CQI Feedback Schemes and Scheduling in LTE," Wireless Communications, IEEE Transactions on, vol. 10, pp. 3436-3448, 2011.

[9] J. Fan, Y. Qinye, G. Y. Li, B. Peng, and Z. Xiaolong, "MCS Selection for Throughput Improvement in Downlink LTE Systems," in Computer Communications and Networks (ICCCN), 2011 Proceedings of 20th International Conference on, 2011, pp. 1-5.

[10] C. Fa-tang and T. Gen-lin, "A novel MCS selection criterion for supporting AMC in LTE system," in Computer Application and System Modeling (ICCASM), 2010 International Conference on, 2010, pp. V6-598-V6-603.

[11] W. Lei, T. Shiauhe, and M. Almgren, "A fading-insensitive performance metric for a unified link quality model," in Wireless Communications and Networking Conference, 2006. WCNC 2006. IEEE, 2006, pp. 2110-2114.

[12] [Online]. http://www.nt.tuwien.ac.at/ltesimulator. 